\title{
Hypoehoic lesions on Transrectal Ultrasound and its correlation to Gleason grade in the diagnosis of Clinically Significant Prostate Cancer: A Prospective Study
}

\author{
Manas Sharma ${ }^{1}$ Rajendra B. Nerli ${ }^{1} \quad$ Sree Harsha Nutalapati ${ }^{1} \quad$ Shridhar C. Ghagane ${ }^{2}$
}

${ }^{1}$ Department of Urology, JN Medical College, KLE Academy of Higher Education and Research, Belagavi, Karnataka, India

2Department of Urology, KLES Kidney Foundation, Urinary Biomarkers Research Centre, KLES Dr. Prabhakar Kore Hospital and Medical Research Centre, Belagavi, Karnataka, India
Address for correspondence Rajendra B. Nerli, MS, MCh (Urology), Professor and Head, Department of Urology, JN Medical College, KLE Academy of Higher Education and Research, JNMC Campus, Belagavi 590010, Karnataka, India

(e-mail: rbnerli@gmail.com).

South Asian J Cancer 2021;10:155-160.

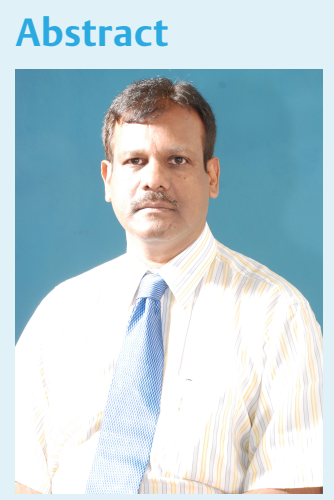

Rajendra B. Nerli

Keywords

- prostate cancer

- transrectal ultrasonography

- prostate biopsy

- hypoechoic lesion

Context The importance of hypoechoic lesions on transrectal ultrasound (TRUS) merits re-assessment in the present era of widespread prostate-specific antigen (PSA) testing.

Aims We aimed to investigate the predictive accuracy of hypoechoic lesions on TRUS of prostate in the diagnosis of prostate cancer and to examine the association of hypoechoic lesions with the aggressiveness of prostate cancer.

Settings and Design This prospective study was conducted in a tertiary care center in South India from November 2017 to December 2019.

Methods and Material We included 151 patients undergoing TRUS-guided 12-core prostate biopsy in view of raised serum PSA with or without suspicious digital rectal examination (DRE) findings in the study. Age, DRE findings, serum PSA level, TRUS findings, and histopathology reports were documented. These were compared between patients with and without hypoechoic lesions on TRUS.

Statistical Analysis Used The statistical analysis for this study was performed using SPSS v20.0 software.

Results Among 151 men, prostate cancer was diagnosed in 68 (45.03\%) with mean age at presentation $69.81 \pm 6.49$ years. Fifty-eight cases $(38.41 \%)$ had hypoechoic lesion on TRUS and the cancer detection rate $(68.96 \%)$ amongst this group was significantly higher than in those without hypoechoic lesion $(p<0.0001)$. Patients with hypoechoic lesion were more likely to have higher grade cancer. Abnormal DRE findings and hypoechoic lesion on TRUS were independent predictors of a clinically significant cancer $(p<0.05)$.

Conclusion Hypoechoic lesion on TRUS can be considered as an indicator of clinically significant prostate cancer.
DOI https://doi.org/10.1055/s-0041-1731906 ISSN 2278-330X

How to cite this article: Sharma M, Nerli R. B, Nutalapati S. H, et al. Hypoehoic lesions on Transrectal Ultrasound and its correlation to Gleason grade in the diagnosis of Clinically Significant Prostate Cancer: A Prospective Study South Asian J Cancer 2021;10(3):155-160. (c) 2021. MedIntel Services Pvt Ltd.

MedIntel Services Pvt Ltd.. This is an open access article published by Thieme under the terms of the Creative Commons Attribution-NonDerivative-NonCommercial-License, permitting copying and reproduction so long as the original work is given appropriate credit. Contents may not be used for commercial purposes, or adapted, remixed, transformed or built upon. (https://creativecommons.org/licenses/by-nc-nd/4.0/). Thieme Medical and Scientific Publishers Private Ltd A-12, Second Floor, Sector -2, NOIDA -201301, India 


\section{Introduction}

Prostate cancer (PCa) was the second most common cancer affecting the elderly male population in the world and the 5 th most common cancer overall as per a 2008 report by Ferlay et al. ${ }^{1}$ The updated data from 2018 showed that PCa surpassed lung cancer and was reported to be the most common cancer in men in 12 regions of the world. It is the second most frequent cause of cancer-specific mortality in five regions. ${ }^{2}$ Due to lack of widespread population-based cancer registries and under-reporting, data regarding the true incidence of PCa in India is inadequate. ${ }^{3}$ As per the Indian Council of Medical Research (ICMR) the incidence rate is nine to ten per $1,00,000$ population. ${ }^{4}$ This incidence rate is higher than that reported from other Asian and African countries, however, lower than the incidence rate reported from the West. PCa contributes to a substantial burden on the health care resources in urban India and its incidence is on the rise even in the rural setting. ${ }^{5}$ Urologists are uniquely proficient by training and experience to perform and interpret transrectal ultrasonography (TRUS) of the prostate. TRUS-guided interventions of the prostate hence are an essential part of urological armamentarium. It is a minimally invasive procedure which enhances patient care and management. ${ }^{6}$ The role of TRUS-guided systematic 12-core prostate biopsy in the diagnosis of PCa is proven beyond doubt. Studies have shown that hypoechoic lesions on TRUS study of prostate have an increased likelihood of harboring a cancerous lesion. ${ }^{7}$ When associated with an abnormal digital rectal examination (DRE) finding and an elevated serum prostate-specific antigen (PSA) level, a hypoechoic lesion on TRUS merits a prostatic biopsy. Since the dawn of PSA era in the early 1990s, a majority of cases of PCa are being diagnosed on the basis of raised PSA levels which is classified as T1c disease. This led to an increase in the detection of so-called clinically insignificant cancers. Epstein et al proposed the following criteria to define clinically insignificant cancer: (1) involvement of less than or equal to one-third of cores positive, $(2) \leq 50 \%$ involvement of any one core, and (3) PSA density $<0.15 \mathrm{ng} / \mathrm{mL}^{2}{ }^{8}$ This was subsequently validated and has proven to be beneficial in making treatment algorithms. ${ }^{9}$ In the present study, we aimed to investigate the predictive accuracy of hypoechoic lesions on TRUS of prostate in the diagnosis of PCa and to examine the association of hypoechoic lesions with the aggressiveness of PCa. Can hypoechoic lesions on TRUS predict the presence of a clinically significant PCa?

\section{Subjects and Methods}

This prospective study was conducted from November 2017 to December 2019 by analyzing the data of patients undergoing TRUS-guided prostate biopsy in view of raised serum PSA level ( $>4 \mathrm{ng} / \mathrm{mL}$ ) with or without suspicious DRE findings. Patients with past history of undergoing prostatic biopsy or diagnosed cases of PCa were excluded from the study. A total of 151 men fulfilled the study criteria and comprised the study group. After obtaining approval (KLES Kidney Foundation Institutional Ethics Committee:
KLESKFIEC/2017/013), informed consent was obtained from study subjects. The clinical parameters such as age, DRE findings, prostate volume, serum PSA, prostate-specific antigen density (PSAD), TRUS findings including hypoechoic lesion, and histopathological examination reports with Gleason grade groups were documented.

The following protocol was followed for TRUS-guided prostate biopsy for all the patients by a single investigator. Patients taking antiplatelet or anticoagulant medications were asked to discontinue the drug as per their treating physician's guidance. Sodium phosphate enema was ordered on the morning of the procedure and a single dose of third generation cephalosporin (injection ceftriaxone $1 \mathrm{~g}$ ) was administered 1 hour prior to the intervention. The patient was placed in left-lateral decubitus position. One ampoule of injection atropine (6 mg) was also administered intravenous (IV) just before starting the procedure. A DRE was performed with adequate lubrication using lignocaine (2\%) jelly. TRUS study of the prostate was performed by Hitachi HI VISION Avius scanner (Hitachi Ltd. Tokyo, Japan) (-Fig. 1A) using 7.5 MHz bi-planar probe. A biopsy guide was attached to the TRUS-probe and 18-guage $\times 18 \mathrm{~cm}$ spring-loaded automatic biopsy needle ( - Fig. 1B) (BARD MAX-CORE Disposable Core biopsy instrument, Bard Peripheral Vascular Inc. Tempe, Arizona, United States) was used to obtain a 12-core prostate biopsy ( - Fig. $2 \mathrm{~B}$ ), each of $22 \mathrm{~mm}$ core length. The samples were submitted for histopathological examination in separate containers with $10 \%$ formalin solution.

\section{Statistical Analysis}

Continuous variables were indicated either as mean \pm standard deviation or median (interquartile range). Categorical variables were reported as number of occurrences and frequency. Statistical comparison of continuous variables was performed using Student $t$-test and Pearson Chi-square test/Fisher exact test was used for comparison of means of
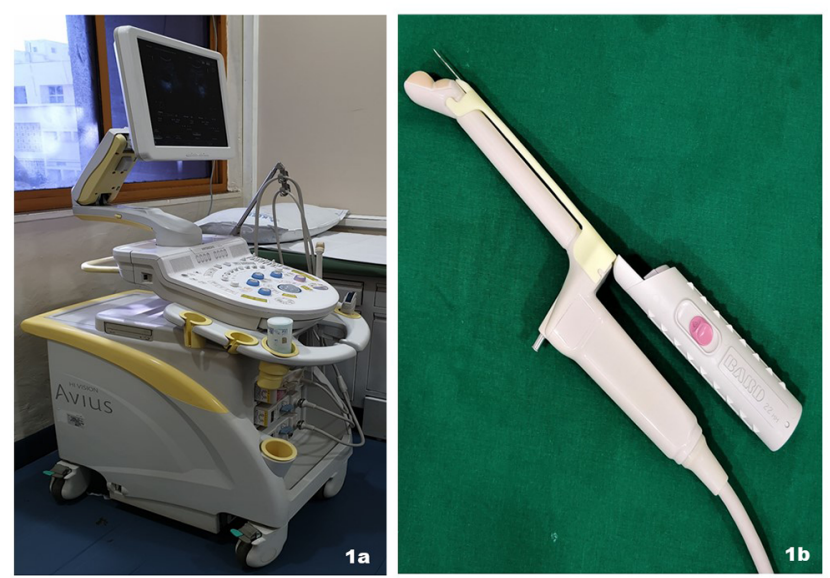

Fig. 1 (a) Hitachi HI VISION Avius ultrasound scanner (Hitachi Ltd. Tokyo, Japan). (b) $7.5 \mathrm{MHz}$ transrectal probe CC531T/CC531L, with a biopsy guide (yellowish white) and an 18-gauge $\times 18 \mathrm{~cm}$ BARD MAX-CORE disposable core biopsy needle (Bard Peripheral Vascular Inc. Tempe, Arizona, United States). 

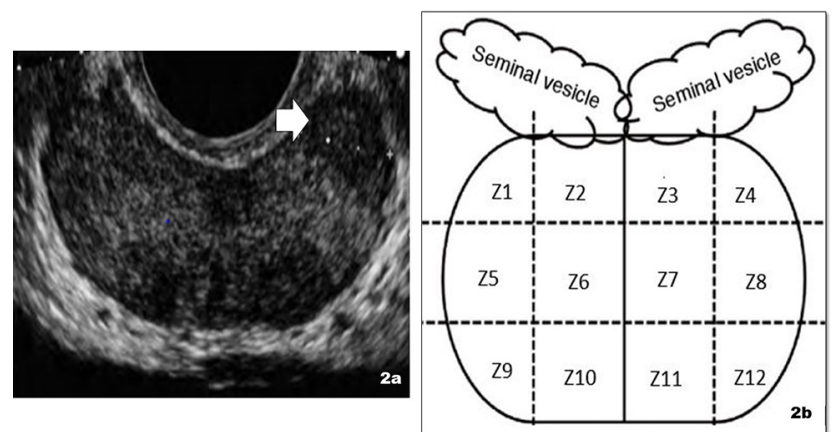

Fig. 2 (a) Transrectal ultrasound image showing a hypoechoic lesion (white arrow) in the peripheral zone (left lobe) of the prostate. (b) Schematic diagram showing template of 12-core biopsy labeled as zones (Z1 to Z12).

categorical variables. The positive and negative predictive values were used to examine the predictive accuracy of TRUS lesions in predicting PCa. The Gleason grades were compared between two groups of CaP patients classified according to presence or absence of hypoechoic lesion on TRUS study. Age, DRE findings, prostate volume (in $\mathrm{mL}$ ), serum PSA, PSAD, and hypoechoic lesion on TRUS were the factors evaluated for risk of harboring clinically significant PCa. Logistic regression analysis of the factors predicting presence of clinically significant PCa was also performed. The outcomes were presented as the odds ratio and $95 \%$ confidence interval based on a two-sided test. All the statistical analyses were performed using SPSS v20.0 software (IBM Corp. Chicago, United States). A $p$-value $<0.05$ was considered as statistically significant.

\section{Results}

Among the 151 men included in the study, 68 (45.03\%) were diagnosed with PCa. The mean age at presentation was $69.81 \pm 6.49$ years with mean and median serum PSA levels were $56.85 \pm 217.23 \mathrm{ng} / \mathrm{mL}$ and $9.62 \mathrm{ng} / \mathrm{mL}$, respectively. Hypoechoic lesion on TRUS imaging ( - Fig. 2A) was noted in 58 patients (38.41\%) and the cancer detection rate amongst them was $68.96 \%$. This was significantly higher than the cancer detection rate in normal TRUS group $(30.10 \%, p<0.0001$ ) ( - Table 1 ). A comparative analysis was performed in 68 patients diagnosed with PCa according to the Gleason Grade Groups ( - Table 2). More number of patients (i.e., $65.78 \%$ ) had hypoechoic lesion on TRUS among the patient with a higher grade cancer (Grade Group $\geq$ III, -Fig. 3) as compared with those with lower Gleason Grade (36.6\%, $p$ <0.05). Patients who were diagnosed with higher grade disease also had significantly older age at presentation, abnormal DRE findings, significantly elevated PSA levels, and $\operatorname{PSAD}(p<0.05)$.

The patients were grouped according to PSA intervals based on presence or absence of PCa on histopathology examination reports. The overall sensitivity, specificity, positive predictive value, negative predictive value, and accuracy of hypoechoic lesion for detecting PCa were 70.59, $87.95,82.76,78.49$, and $80.13 \%$, respectively. The predictive efficacy also varied amongst different PSA intervals, showing a trend toward increasing accuracy in the higher PSA interval (>20 ng/mL) (-Table 3). Similarly, the relationship between hypoechoic lesion and Gleason Grade group in different PSA intervals was presented (-Table 4). The existence of hypoechoic lesion on TRUS was significantly associated with higher grade cancer in patient with PSA interval of $>20 \mathrm{ng} / \mathrm{mL}(p<0.05)$. Simple logistic regression analysis of the factors predicting presence of clinically significant PCa in PCa patients revealed that age, abnormal DRE findings, prostate volume, serum PSA, PSAD, and hypoechoic lesion on TRUS were significant factors $(p<0.05)$, whereas, abnormal DRE findings (nodule on DRE or hard consistency of prostate) and hypoechoic lesion on TRUS were identified as significant predictors of clinically significant cancer on multiple logistic regression analysis $(p<0.05)$ ( - Table 5$)$.

Table 1 Clinical characteristics of the study group and differences between the groups based on transrectal ultrasound findings

\begin{tabular}{|c|c|c|c|c|}
\hline Variable & $\begin{array}{l}\text { All cases } \\
(n=151)\end{array}$ & $\begin{array}{l}\text { TRUS without visible } \\
\text { lesion }(n=93)\end{array}$ & $\begin{array}{l}\text { Hypoechoic lesion on TRUS } \\
(n=58)\end{array}$ & $p$-Value \\
\hline Age (years) & $69.81 \pm 6.49$ & $68.09 \pm 7.93$ & $71.12 \pm 7.88$ & 0.0235 \\
\hline Abnormal DRE & $35(23.17)$ & $12(12.90)$ & $23(39.65)$ & 0.0002 \\
\hline Prostate volume (cc) & $57.09 \pm 19.13$ & $56.72 \pm 15.12$ & $57.26 \pm 20.61$ & 0.85 \\
\hline Serum PSA & $56.85 \pm 217.23$ & $22.05 \pm 68.38$ & $134.79 \pm 365.5$ & 0.004 \\
\hline Median serum PSA & $9.62(6.1-19.1)$ & $7.21(5.1-13.3)$ & $18.94(12.5-64.8)$ & $<0.0001$ \\
\hline PSA density & $0.17(0.09-0.35)$ & $0.12(0.07-0.24)$ & $0.32(0.17-1.11)$ & $<0.0001$ \\
\hline $\mathrm{BPH}$ & $83(54 \%)$ & 65 (69.9\%) & $18(31.04 \%)$ & $<0.0001$ \\
\hline Ca prostate & $68(46.03 \%)$ & $28(30.10 \%)$ & $40(68.96 \%)$ & $<0.0001$ \\
\hline \multicolumn{5}{|l|}{ Gleason grade } \\
\hline Group I/II & 30 & 20 & 10 & \multirow[t]{2}{*}{0.0039} \\
\hline Group $\geq$ III & 38 & 12 & 26 & \\
\hline
\end{tabular}

Abbreviations: DRE, digital rectal examination; BPH, benign prostatic hyperplasia; PSA, prostate-specific antigen; TRUS, transrectal ultrasound. Note: Values are presented as mean \pm SD, number (\%), or median (interquartile range). 
Table 2 Clinical characteristics of patients with prostate cancer ( $n=68)$ according to Gleason grade groups

\begin{tabular}{|l|l|l|l|}
\hline Variable & $\begin{array}{l}\text { Gleason grade Group I/II } \\
(\boldsymbol{n}=30)\end{array}$ & $\begin{array}{l}\text { Gleason grade Group } \geq \text { III } \\
(\boldsymbol{n}=38)\end{array}$ & $p$-Value \\
\hline Age (years) & $65.52 \pm 6.94$ & $70.48 \pm 4.88$ & 0.001 \\
\hline Abnormal DRE (\%) & $10(33.33 \%)$ & $24(63.15 \%)$ & 0.015 \\
\hline Prostate volume (cc) & $54.47 \pm 7.87$ & $59.55 \pm 6.97$ & 0.006 \\
\hline Serum PSA (ng/mL) & $10.14(4.80-20.46)$ & $40.88(12.48-108.97)$ & 0.001 \\
\hline PSA Density & $0.22(0.14-0.47)$ & $0.96(0.38-2.84)$ & 0.001 \\
\hline Hypoechoic lesion on TRUS & $11(36.66)$ & $25(65.78)$ & 0.017 \\
\hline
\end{tabular}

Abbreviations: DRE, digital rectal examination; PSA, prostate-specific antigen; TRUS, transrectal ultrasound.

Note: Values are presented as mean \pm SD, number (\%), or median (interquartile range).

Table 3 Predictive efficacy of hypoechoic lesion for prostate cancer in different PSA intervals

\begin{tabular}{|c|c|c|c|c|c|c|c|c|c|}
\hline $\begin{array}{l}\text { PSA } \\
\text { interval }\end{array}$ & & $\begin{array}{l}\text { CaP } \\
(n=68)\end{array}$ & $\begin{array}{l}\text { ВРH } \\
(n=83)\end{array}$ & $\begin{array}{l}\text { Sensitivity } \\
\text { (\%) }\end{array}$ & $\begin{array}{l}\text { Specificity } \\
\text { (\%) }\end{array}$ & $\begin{array}{l}\text { PPV } \\
(\%)\end{array}$ & $\begin{array}{l}\text { NPV } \\
(\%)\end{array}$ & $\begin{array}{l}\text { Accuracy } \\
(\%)\end{array}$ & $p$-Value \\
\hline \multirow[t]{2}{*}{$\begin{array}{l}4-10 \mathrm{ng} / \\
\mathrm{mL}\end{array}$} & $\begin{array}{l}\text { Hypoechoic } \\
\text { lesion }\end{array}$ & 10 & 5 & 66.67 & 76.19 & 66.67 & 76.19 & 72.22 & 0.01 \\
\hline & $\begin{array}{l}\text { No visible } \\
\text { lesion }\end{array}$ & 5 & 16 & & & & & & \\
\hline \multirow[t]{2}{*}{$\begin{array}{l}>10-20 \\
\mathrm{ng} / \mathrm{mL}\end{array}$} & $\begin{array}{l}\text { Hypoechoic } \\
\text { lesion }\end{array}$ & 17 & 3 & 62.96 & 86.36 & 85.00 & 65.52 & 73.47 & 0.0004 \\
\hline & $\begin{array}{l}\text { No visible } \\
\text { lesion }\end{array}$ & 10 & 19 & & & & & & \\
\hline \multirow[t]{2}{*}{$\begin{array}{l}>20 \mathrm{ng} / \\
\mathrm{mL}\end{array}$} & $\begin{array}{l}\text { Hypoechoic } \\
\text { lesion }\end{array}$ & 21 & 2 & 80.77 & 95.00 & 91.30 & 88.37 & 89.39 & $<0.0001$ \\
\hline & $\begin{array}{l}\text { No visible } \\
\text { lesion }\end{array}$ & 5 & 38 & & & & & & \\
\hline
\end{tabular}

Abbreviations: BPH, benign prostatic hyperplasia; CaP, cancer prostate; NPV, negative predictive value; PPV, positive predictive value; PSA, prostate-specific antigen.
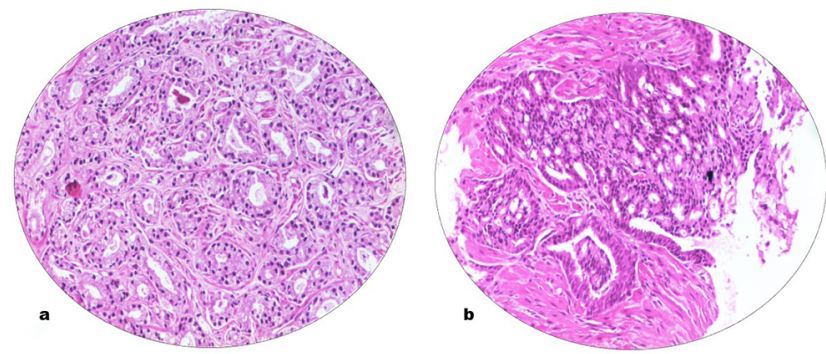

Fig. 3 (a) $\mathrm{H}$ \& E staining 40x, showing Gleason pattern 3. Well formed, individual glands of varying sizes. (b) H\&E staining 40x, showing Gleason pattern 4. Cribriform pattern, neoplastic cells running across the core.

\section{Discussion}

PCa incidence in the Indian subcontinent is still lower as compared with the western countries. ${ }^{3}$ However, the incidence is on the rise perhaps due to stressful lifestyle, increased public awareness, and the misuse of serum PSA testing during general health check-up of middle aged and elderly men. The PSA threshold for subjecting a patient to prostatic biopsy is a topic of widespread dispute. At present, it is clear that different populations need to have a different cut-off levels of PSA. According to Prostate Cancer Screening trial (PLCO study) in the United States, a PSA level $\geq 4 \mathrm{ng} / \mathrm{mL}$ was used as a threshold for biopsy, ${ }^{10}$ whereas European Prostate Cancer Screening Trial (ERSPC) had set a PSA level of $\geq 3.0 \mathrm{ng} / \mathrm{mL}$ as a trigger for biopsy. ${ }^{11}$ These recommendations led to an unnecessarily high number of prostatic biopsies and an increase in the diagnosis of clinically insignificant PCa. In a study from India, Dubey questioned the routine use of serum PSA testing and advocated against it owing to a low incidence of PCa in India. ${ }^{12}$ The author appropriately states that if a patient requests PSA testing, he should be informed about its benefits and harms and shared decision should be taken in the patients' best interest. We would like to differ with this report as we have noticed a rise in the incidence of PCa in our own clinical experience. ${ }^{5}$ This can possibly be attributed to the improvement in health care availability and better imaging modalities.

Once the decision to perform a biopsy is made, TRUS-guided biopsy is given consideration. It allows visualization of the prostate and suspicious lesions which can then be targeted for biopsy under real-time guidance. ${ }^{13}$ Onur et al reported the contemporary impact of TRUS in the diagnosis of PCa; they found $25.5 \%$ cancer detection rate in targeted biopsy of hypoechoic lesions. ${ }^{14}$ Similarly, Toi et al described that biopsy obtained from a lesion on TRUS was two times more likely to reveal malignancy when compared with normal TRUS imaging biopsy sample. ${ }^{15}$ Several studies in literature support the role of biopsy performed from a visible 
Table 4 Association between hypoechoic lesion and Gleason grade groups in different PSA intervals

\begin{tabular}{|l|l|l|l|l|}
\hline PSA interval & TRUS Finding & Grade I/II $(\boldsymbol{n}=\mathbf{3 0})$ & Grade $\geq$ III $(\boldsymbol{n}=38)$ & $\boldsymbol{p}$-Value \\
\hline \multirow{3}{*}{$4-10 \mathrm{ng} / \mathrm{mL}$} & Hypoechoic lesion & 3 & 2 & $>0.05$ \\
\cline { 2 - 5 } & No visible lesion & 4 & 1 & $>0.05$ \\
\hline$>10-20 \mathrm{ng} / \mathrm{mL}$ & Hypoechoic lesion & 4 & 5 & \\
\cline { 2 - 5 } & No visible lesion & 6 & 4 & 0.029 \\
\hline$>20 \mathrm{ng} / \mathrm{mL}$ & Hypoechoic lesion & 4 & 8 & 18 \\
\cline { 2 - 5 } & No visible lesion & 9 & 8 & \\
\hline
\end{tabular}

Table 5 Logistic regression analysis of the factors predicting presence of clinically significant prostate cancer (Gleason Grade Group $\geq$ III) in 68 patients

\begin{tabular}{|l|l|l|l|l|l|l|}
\hline \multirow{2}{*}{ Variables } & \multicolumn{3}{|c|}{ Simple logistics regression } & \multicolumn{3}{c|}{ Multiple logistics regression } \\
\cline { 2 - 7 } & OR & $95 \% \mathrm{Cl}$ & $\boldsymbol{p}$-Value & OR & $95 \% \mathrm{Cl}$ & $p$-Value \\
\hline Age & 1.47 & $1.45-1.49$ & 0.001 & & & 0.001 \\
\hline Abnormal DRE & 5.88 & $3.87-8.94$ & 0.001 & 2.98 & $1.69-4.97$ & \\
\hline Prostate volume & 0.98 & $0.97-0.99$ & 0.004 & & & \\
\hline Serum PSA & 1.05 & $1.04-1.06$ & $<0.001$ & & & \\
\hline PSA density & 6.48 & $4.57-9.87$ & 0.001 & & & $1.34-2.67$ \\
\hline Hypoechoic lesion on TRUS & 4.14 & $3.18-6.12$ & $<0.001$ & 1.87 & 0.028 \\
\hline
\end{tabular}

Abbreviations: $\mathrm{Cl}$, confidence interval; DRE, digital rectal examination; OR, odds ratio; PSA, prostate-specific antigen; TRUS, transrectal ultrasound.

lesion on TRUS to increase the yield and reduce the rate of false negative biopsies. In our study, 58 patients (38.41\%) had presence of hypoechoic lesion on TRUS and the cancer detection rate amongst them was $68.96 \%$. This was significantly higher than the rate in normal TRUS imaging group (30.10\%, $p<0.0001)$. This is in consensus with the cancer detection rate reported in literature (62.9-67.2\%).

We must highlight that not all hypoechoic lesions on TRUS imaging suggest the presence of malignancy, contrariwise, not all cancerous lesions appear hypoechoic. It is known that approximately 60 to $70 \%$ of PCa lesions appear hypoechoic on TRUS; the rest may be isoechoic $(30-40 \%)^{16}$ or rarely hyperechoic $(<2 \%)^{17,18}$ Presence of hypoechoic lesions in the prostate can also be noted in case of prostatic lymphoma, granulomatous prostatitis, and rarely prostatic infarction. ${ }^{19}$ Some reports suggest that a limited sextant biopsy (six cores) could also be sufficient provided that the hypoechoic areas if present, are adequately sampled. ${ }^{20}$ However, there is now a clear consensus that a sextant biopsy is insufficient; a 10or 12-core biopsy should be routinely employed including the targeting of suspicious lesions on TRUS..$^{21}$ We performed a systematic 12-core prostate biopsy including a core from suspicious hypoechoic lesion.

Patients who had hypoechoic lesions in our series were also found to have an aggressive disease. There were 38 patients with high-grade tumor (Gleason grade group $\geq$ III) of which $25(65.78 \%)$ cases had hypoechoic lesions. Similar findings were reported by Noh et al, as $64.2 \%$ of their patients in the higher Gleason group (Gleason score >7) were found to have hypoechoic lesion on TRUS. ${ }^{22}$ To evaluate the relationship between cancer and hypoechoic lesion, we also subdivided the patients as per their PSA levels. This revealed that hypoechoic lesions were associated with PCa among all the PSA intervals with the highest predictive efficacy in cases with PSA levels $>20 \mathrm{ng} / \mathrm{mL}$. Patients with highest PSA levels $(>20 \mathrm{ng} / \mathrm{mL}$ ) were also found have significant association with occurrence of high-grade tumor $(p<0.05)$. Similar findings were reported by Yang et al. ${ }^{23}$ Numerous investigators have presented that abnormal DRE findings were associated with aggressive cancer ${ }^{24-26}$; our study also confirmed these findings. Nodules on DRE or hard consistency of prostate was independent variable associated with high-grade cancer $(p<0.05)$.

There have been arguments about the benefits and limitations of TRUS even though it is currently being widely utilized for the diagnosis of PCa. TRUS carries poor accuracy in the detection of small lesions, is highly operator dependent, and cannot be applied for local staging, detection of extracapsular spread, and seminal vesicle infiltration. ${ }^{27}$ Multiparametric MRI hence acts as an important tool which is now being increasingly utilized in the diagnosis of PCa. It has a high sensitivity and specificity for cancer staging, 91 and 96\%, respectively. ${ }^{6}$ The introduction of MRI-guided biopsy has altered the paradigm regarding prostate biopsies. Even though it has similar overall PCa detection rates compared with TRUS-guided biopsies, it has higher detection rate of clinically significant $\mathrm{PCa} .^{28}$ In spite of this, TRUS continues to play a key role in PCa diagnosis. It provides dynamic imaging, has virtually no contraindications, and is less time consuming. It also imparts less financial burden on patients which makes it an attractive option suitable for our country.

The results of the present study are promising; however, they are not without limitations. The primary limitation to generalization of our results is a single institutional study 
with a relatively small study cohort. It can be addressed in future research. Inclusion of factors like number of biopsy cores positive for malignancy and the percentage of core involved can help us identify clinically significant PCa in a more objective manner. This can act as a valuable tool in making treatment-related decisions.

\section{Conclusion}

Diagnosis of PCa has experienced a stage migration due to extensive use of serum PSA testing. It raises the concern about the overdiagnosis of clinically insignificant cancers. Patients with prostate cancer harboring a hypoechoic lesion on TRUS had more aggressive disease as compared with those without any abnormal findings. Hypoechoic lesion can improve the predictive efficacy of diagnosis of prostate cancer based on different PSA intervals. We conclude that hypoechoic lesion on TRUS can be considered as an indicator of clinically significant prostate cancer in the background of suspicious DRE findings and elevated serum PSA.

\section{Conflict of Interest}

None declared.

\section{References}

1 Ferlay J, Shin HR, Bray F, Forman D, Mathers C, Parkin DM. Estimates of worldwide burden of cancer in 2008: GLOBOCAN 2008. Int J Cancer 2010;127(12):2893-2917

2 Ferlay J, Colombet M, Soerjomataram I, et al. Estimating the global cancer incidence and mortality in 2018: GLOBOCAN sources and methods. Int J Cancer 2019;144(8):1941-1953

3 Hariharan K, Padmanabha V. Demography and disease characteristics of prostate cancer in India. Indian J Urol 2016;32(2):103-108

4 E.H. News Bureau. ICMR releases data on incidence rate of prostate cancer in India. Express Health Care. 2019. Available at: https://www.expresshealthcare.in/news/icmr-releasesdata-on-incidence-rate-of-prostate-cancer-in-india/414265. Accessed April 2, 2020

5 Ghagane SC, Nerli RB, Hiremath MB, Wagh AT, Magdum PV. Incidence of prostate cancer at a single tertiary care center in North Karnataka. Indian J Cancer 2016;53(3):429-431

6 Harvey CJ, Pilcher J, Richenberg J, Patel U, Frauscher F. Applications of transrectal ultrasound in prostate cancer. $\mathrm{Br}$ J Radiol 2012;85(Spec No 1) :S3-S17

7 Gosselaar C, Roobol MJ, Roemeling S, Wolters T, van Leenders GJ, Schröder FH. The value of an additional hypoechoic lesion-directed biopsy core for detecting prostate cancer. BJU Int 2008;101(6):685-690

8 Epstein JI, Walsh PC, Carmichael M, Brendler CB. Pathologic and clinical findings to predict tumor extent of nonpalpable (stage T1c) prostate cancer. JAMA 1994;271(5):368-374

9 Bastian PJ, Mangold LA, Epstein JI, Partin AW. Characteristics of insignificant clinical T1c prostate tumors. A contemporary analysis. Cancer 2004;101(9):2001-2005

10 Andriole GL, Crawford ED, Grubb RL III, et al; PLCO Project Team. Mortality results from a randomized prostate-cancer screening trial. N Engl J Med 2009;360(13):1310-1319

11 Roobol MJ, Kranse R, Bangma CH, et al; ERSPC Rotterdam Study Group. Screening for prostate cancer: results of the Rotterdam section of the European randomized study of screening for prostate cancer. Eur Urol 2013;64(4):530-539

12 Dubey D. The routine use of prostate-specific antigen for early detection of cancer prostate in India: is it justified? Indian J Urol 2009;25(2):177-184

13 Heijmink SW, van Moerkerk H, Kiemeney LA, Witjes JA, Frauscher F, Barentsz JO. A comparison of the diagnostic performance of systematic versus ultrasound-guided biopsies of prostate cancer. Eur Radiol 2006;16(4):927-938

14 Onur R, Littrup PJ, Pontes JE. Bianco FJ Jr. Contemporary impact of transrectal ultrasound lesions for prostate cancer detection. J Urol 2004;172(2):512-514

15 Toi A, Neill MG, Lockwood GA, Sweet JM, Tammsalu LA, Fleshner NE. The continuing importance of transrectal ultrasound identification of prostatic lesions. J Urol 2007;177(2):516-520

16 Kuligowska E, Barish MA, Fenlon HM, Blake M. Predictors of prostate carcinoma: accuracy of gray-scale and color Doppler US and serum markers. Radiology 2001;220(3):757-764

17 Egawa S, Wheeler TM, Greene DR, Scardino PT. Unusual hyperechoic appearance of prostate cancer on transrectal ultrasonography. Br J Urol 1992;69(2):169-174

18 Malik R, Pandya VK, Naik D. Transrectal ultrasonography for evaluation of various benign and malignant prostatic lesions and their histopathological correlation. Indian $\mathrm{J}$ Radiol Imaging 2004;14:155-157

19 Alan JW, Louis RK, Alan WP, Craig AP, Campbell-Walsh Urology. 11th ed. Elsevier; 20162582

20 Xu G, Yao M, Wu J, et al. Diagnostic value of different systematic prostate biopsy methods in the detection of prostate cancer with ultrasonographic hypoechoic lesions-a comparative study. Urol Int 2015;95(2):183-188

21 Nesrallah L, Nesrallah A, Antunes AA, Leite KR, Srougi M. The role of extended prostate biopsy on prostate cancer detection rate: a study performed on the bench. Int Braz J Urol 2008;34(5):563-570, discussion 570-571

22 Noh TI, Shin YS, Shim JS, et al. Are hypoechoic lesions on transrectal ultrasonography a marker for clinically significant prostate cancer? Korean J Urol 2013;54(10):666-670

23 Yang T, Zhang L, Chen Y, Cai Y, Jiang H, Ding Q. The predictive efficacy of hypoechoic lesion in ultrasound for prostate cancer in Chinese people: five-year experience in a moderated 10-core transperineal prostate biopsy procedure. Oncotarget 2017;8(45):79433-79440

24 Gosselaar C, Roobol MJ, Roemeling S, van der Kwast TH, Schröder FH. Screening for prostate cancer at low PSA range: the impact of digital rectal examination on tumor incidence and tumor characteristics. Prostate 2007;67(2):154-161

25 Gosselaar C, Roobol MJ, Roemeling S, Schröder FH. The role of the digital rectal examination in subsequent screening visits in the European randomized study of screening for prostate cancer (ERSPC), Rotterdam. Eur Urol 2008;54(3):581-588

26 Okotie OT, Roehl KA, Han M, Loeb S, Gashti SN, Catalona WJ. Characteristics of prostate cancer detected by digital rectal examination only. Urology 2007;70(6):1117-1120

27 Patel U, The prostate and seminal vesicles. In: Allan P, Baxter G, Weston M, eds. Clinical Ultrasound. 3rd ed. Edinburgh: Churchill-Livingstone; 2011 572-592

28 Wegelin O, van Melick HH, Hooft L, et al. Comparing three different techniques for magnetic resonance imaging-targeted prostate biopsies: a systematic review of in-bore versus magnetic resonance imaging-transrectal ultrasound fusion versus cognitive registration. is there a preferred technique? Eur Urol 2017;71(4):517-531 